\title{
Double Crossover Points in the DFA of Heartbeats in Patients with Sleep Apnea
}

\author{
Midori Ichimaru' ${ }^{1}$, Yuhei Ichimaru' ${ }^{2}$, George B. Moody ${ }^{3}$, Kuniaki Otsuka ${ }^{1}$ \\ ${ }^{1}$ Department of Medicine, Tokyo Women's Medical University, Medical Center East, Tokyo, Japan; ${ }^{2}$ Department of Clinical Nutri- \\ tion and Informatics, Tokyo Kasei University, Tokyo, Japan; ${ }^{3}$ Harvard-MIT Division of Health Science and Technology, Boston, \\ USA. \\ Email: ichimi6174@hotmail.co.jp
}

Received September $10^{\text {th }}, 2012$; revised October $20^{\text {th }}, 2012$; accepted November $19^{\text {th }}, 2012$

\begin{abstract}
Cardiovascular and cerebrovascular events have been observed during night-time associated with periodic breathing including sleep apnea and Cheyne-Stokes respiration. Early detection and treatment is important to reduce night-time events. We clarified the characteristics of the dynamic nature of heartbeats associated with periodic breathing by using detrended fluctuation analysis (DFA). We analyzed heartbeats in eight recordings from the MIT-BIH Polysomnographic database. We observed two crossover points and defined three scaling exponents, $\beta 1$ ( $\mathrm{n} \leq 40$ beats), $\beta 2(50 \leq \mathrm{n}$ $\leq 200)$, and $\beta 3(251 \leq \mathrm{n} \leq 1584)$. Compared with $\beta 1(1.21 \pm 0.13)$ and $\beta 3(0.92 \pm 0.16)$, scaling exponent $\beta 2(0.62 \pm$ $0.16)$ showed the statistically lowest value $(\mathrm{p}<0.05)$. And there was a negative relationship between the scaling exponent $\beta 2$ and apnea/hypopnea index $(\mathrm{p}<0.05)$. These results indicate that DFA analysis of heartbeats may be useful for the early detection of sleep associated breathing disorders including sleep apnea and its severity.
\end{abstract}

Keywords: Sleep Apnea; DFA (Detrended Fluctuation Analysis); Scaling Exponent; Crossover Points; Heartbeats

\section{Introduction}

Sleep is not a passive state but an active neurophysiological condition, and sometimes a state of heightened autonomic activity is associated with REM sleep or sleep apnea. Life-threatening arrhythmias and cerebrovascular events have been observed during night-time [1-4]. Epidemiological studies suggest that hypertension is frequently observed in patients with sleep apnea syndrome [5-14]. Early diagnosis or treatment of sleep apnea is thus important to reduce night-time cardiovascular events. Sleep disordered breathing including sleep apnea syndrome is usually diagnosed by polysomnographic techniques. This procedure takes a lot of time, requires instruments to monitor several physiological signals, needs specific skills and is usually performed by specially trained technicians in sleep laboratories. The diagnosis of sleep-disordered breathing is sometimes missed because of the lack of a simple and adequate screening tool [15]. Use of 24-hour electrocardiogram and analysis of heart rate may be useful as a "rule out test" for sleep disorder breathing in patients with congestive heart failure. During the events of periodic breathing including CheyneStokes respiration and obstructive sleep apnea, a cyclic variation of the heart rate is observed [16]. Spectral analysis of the heart rate obtained from 24-hour electro- cardiographic monitoring can afford useful information concerning periodic breathing. The peak value in the very low frequency band (VLF: $0.01-0.05 \mathrm{~Hz}$ ) is associated with the presence of periodic breathing or CheyneStokes respiration $[17,18]$. Thus, the analysis of nocturnal heart rate variability by spectral analysis is a quick and easy screening tool for sleep-disordered breathing [19]. Detrended fluctuation analysis (DFA) has become a widely-used method to detect fluctuations that cannot be distinguished by linear analysis methods [20]. Analysis of the fractal characteristics of heartbeats yields more powerful prognostic information than traditional measurement of heart rate variability in patients with heart failure [20-22]. DFA has been applied to evaluate patients with sleep apnea syndrome [23]. In extracting a scaling exponent $\alpha 2$, a crossover point was set at 70 beats. In this study, we conducted DFA of heartbeats in subjects with sleep apnea by setting two crossover points and evaluated whether scaling exponents have an ability to evaluate the relationship between the number of apnea/hypopnea events and the value of the scaling exponent.

\section{Methods}

We selected 8 subjects from the MIT-BIH Polysomno- 
graphic Database, which we had previously developed [24]. These subjects underwent night-time polysomnography with ECG (electro-cardiogram), EEG (electroencephalogram), nasal and oral air flow, respiratory movement, and systemic blood pressure. These signals were digitized at a sampling interval of $250 \mathrm{~Hz}$. Each heartbeat was detected automatically by using the ARISTOTLE arrhythmia detector (developed by G.B. Moody in MIT), and each beat annotation was verified by visual inspection and re-edited by cursor in the screen editor (WAVE). In the MIT-BIH polysomnographic database, nasal and thoracic respiratory signals in patients with sleep apnea were included. These sleep recordings were evaluated by a sleep expert at Beth-Israel Hospital who followed the guidelines of Rechtschaffen and Kales [25]. The number of sleep apnea and hypopnea events was measured by observing the respiratory signals with cessation of respiration for at least 10 seconds or a hypopnea in which airflow decreased by $50 \%$ for 10 seconds. We used the detrended fluctuation analysis method (DFA), which had been developed to characterize the correlation properties in non-stationary time series of a physiological signal [20]. We have developed a DFA analysis program by using VBA (Visual Basic for Applications) and Microsoft Excel according to the DFA algorithm [20]. As a first step, the inter-beat interval time series is integrated and $\mathrm{Y}(\mathrm{k})=\left(\right.$ for $\mathrm{i}=1$ to $\mathrm{k}$; $(\mathrm{B}(\mathrm{i})-\mathrm{B} a v e)^{2}$; next $\left.\mathrm{i}\right)$ is obtained; where Bave is the average instantaneous RRinterval signal, and B(i) is the beat-to-beat signal. The integrated time series is divided into boxes. For each box of length n, a least squares line (the "trend") is fit to the $\mathrm{n}$ samples in the box. The fluctuations within each box are the differences between the samples and the fitted, and the root-mean-square fluctuation over all boxes of size $n$ is denoted as $F(n)$. This calculation is repeated for all values of $n$ between 3 and the length of the time series. After that, for each $n$ size, $F(n)$ is obtained. The plot of $\log _{10}(\mathrm{~F}(\mathrm{n}))$ against $\log _{10}(\mathrm{n})$ was obtained. The slope of the line relating $F(n)$ and $n$ in a $\log -\log$ scale is the scaling exponent $\alpha$. Traditionally a DFA plot consists of two distinct regions characterized by two straight lines before and after a crossover point (CP). In our study, the scaling exponent $\alpha 1(3 \leq \mathrm{n} \leq 16)$, and $\alpha 2(17 \leq \mathrm{n} \leq 64)$ are calculated. As we observed two crossover points, we defined an additional three scaling exponents, $\beta 1, \beta 2$ and $\beta 3$. The values of $\beta 1, \beta 2$, and $\beta 3$ correspond to the slopes of $\log _{10} \mathrm{~F}(\mathrm{n})$ as a function of $\log _{10}(\mathrm{n})$ in the range between $3 \leq \mathrm{n} \leq 40$ beats, $50 \leq \mathrm{n} \leq$ 200 beats, and $251 \leq \mathrm{n} \leq 1584$ beats, respectively. The range of $\beta 1$ consisted of 13 scales, $\beta 2$ involved 7 scales and $\beta 3$ involved 9 scales.

\section{Statistical Analysis}

All data are present as mean \pm standard deviation. The
Bartlett multiple-sample test was performed for equal variances. Analysis of variance (ANOVA) for repeated measurements was applied to examine significant changes in the measured variables of exponents $\beta 1, \beta 2$, and $\beta 3$. The paired t-test was used to examine the differences. Probability levels $<0.05$ were considered significant.

\section{Results}

The scaling behavior in a patient with sleep apnea (total number of sleep apnea events: 264) demonstrates at least two crossover points (Figure 1). The box size of the first crossover point is 40 beats, and that of the second one is 251 beats. Scaling exponents obtained by the slopes of fitted lines were as follows; $\alpha 1=1.38, \alpha 2=0.98, \beta 1=$ $1.35, \beta 2=0.44$, and $\beta 3=1.06$.

The scaling behavior in a subject with a sleep apnea index of less than 5 events/hour shows no remarkable change before and after the crossover points (Figure 2: $\alpha 1=1.23, \alpha 2=1.02, \beta 1=0.997, \beta 2=0.84, \beta 3=1.005)$.

To examine whether there is a difference in the scaling exponents, we performed a statistical analysis on eight subjects. A significantly lower value was obtained in the scaling exponent of $\alpha 2(1.03 \pm 0.08)$ compared with the scaling exponent of $\alpha 1(1.23 \pm 0.11)$. The mean and standard deviation of scaling exponents of $\beta 1=1.21 \pm 0.13$, $\beta 2=0.62 \pm 0.18$ and $\beta 3=0.92 \pm 0.16$ were found and statistically significant changes are observed $(\mathrm{p}<0.05)$, with $\beta 2$ being the lowest value (Figure 3).

As shown in Figure 4, the relationship between the numbers of sleep apnea/hypopnea index and the values

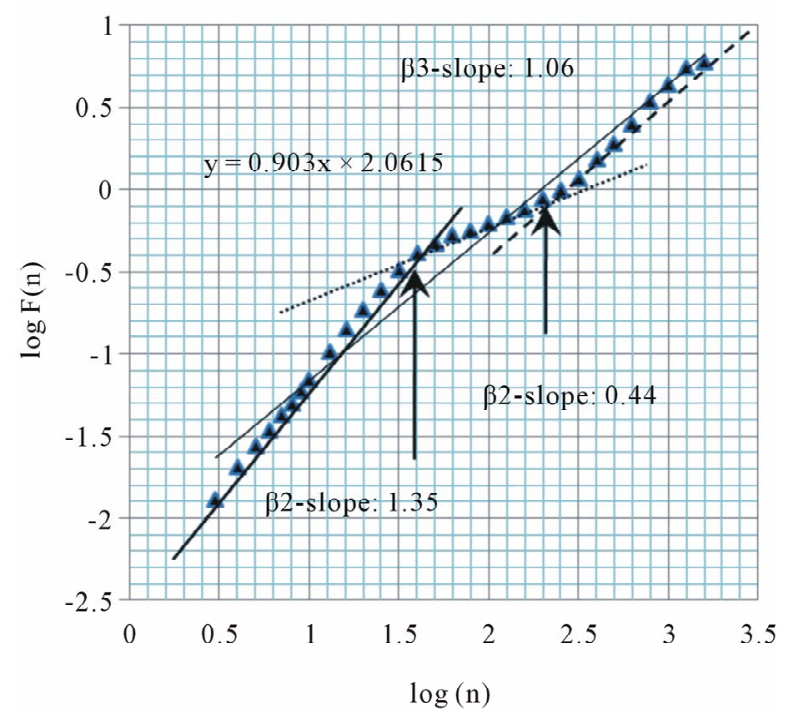

Figure 1. Plot of scaling function $\log (F(n))$ versus $\log (n)$ for the heartbeats using DFA from MIT-BIH Polysomnographic Database (SLP48: A subject with sleep apnea syndrome: apnea/hypopnea index 46.8). Arrows indicate "crossover" points, which we hypothesized. We calculated the slope of the plot between the crossover points, and defined it as $\beta 2$. 


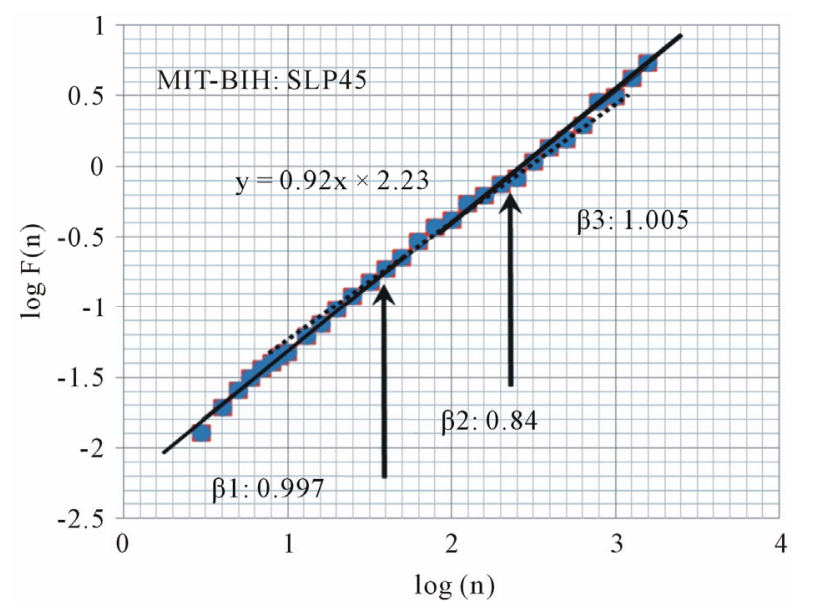

Figure 2. Plot of scaling function $\log (F(n))$ versus $\log (n)$ for the heart beat using DFA from MIT-BIH Polysomnographic Database (SLP45: A subject with low apnea/hypopnea index: 5). The slope of the regression line is 0.92 . Arrows indicate "crossover" points, which we hypothesized.

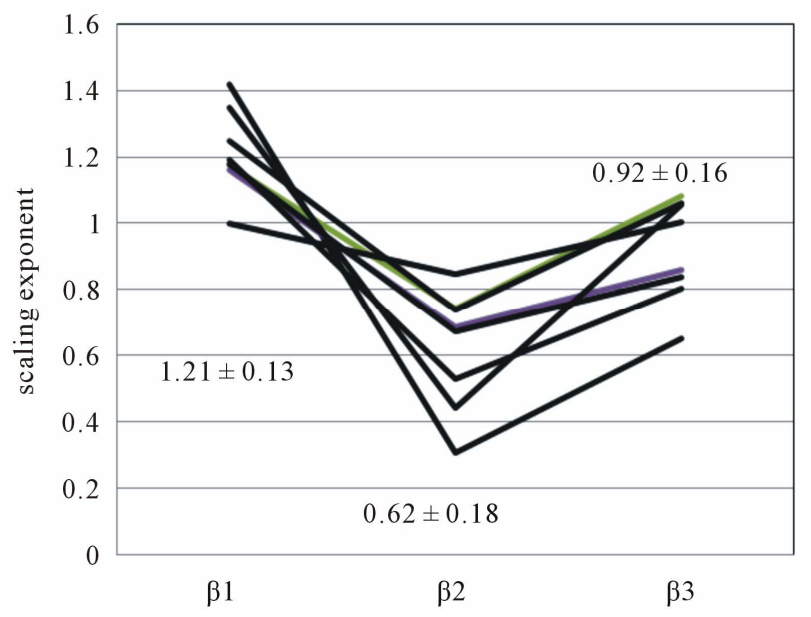

Figure 3. Scaling exponent $\beta 1, \beta 2, \beta 3$ in eight subjects.

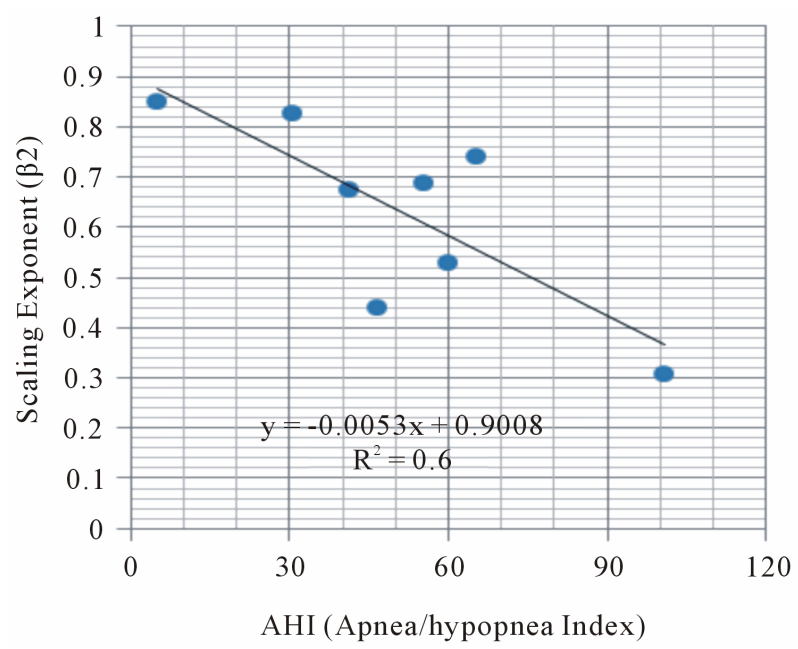

Figure 4. Relationship between sleep apnea/hypopnea events (AHI) and scaling exponent. of the $\beta 2$ exponent in eight subjects showed the negative correlation $(\mathrm{p}<0.05)$.

\section{Discussion}

In this study, we analyzed the scaling behavior in heartbeats by using the DFA method. There were apparent two crossover points in the plot of $\log \mathrm{F}(\mathrm{n})$ and $\log (\mathrm{n})$ derived from the DFA method in subjects suffering from sleep apnea syndrome. We detected a statistically significant decrease in the $\beta 2$ value in subjects with sleep apnea syndrome and a negative correlation between numbers of sleep apnea events and $\beta 2$ values.

Penzel et al. determined two scaling exponents, $\alpha 1$ and $\alpha 2$ instead of using the traditional crossover point [23]. They defined exponent $\alpha 1$ as the slope of $\log \mathrm{F}(\mathrm{t})$ as a function of logt in the range $10 \leq \mathrm{t} \leq 40$ beats, and $\alpha 2$ as the slope in the range $70 \leq \mathrm{t} \leq 300$ beats. We defined exponent $\beta 2$ as the slope in the range $50 \leq \mathrm{n} \leq 200$ beats. This means the scaling exponent $\alpha 1, \alpha 2$ as defined by Penzel and the scaling exponent $\beta 1, \beta 2$ as defined by us covers almost the same range. They reported that the average scaling exponents of $\alpha 2$ value obtained from all night data in healthy $(\mathrm{H})$, moderate sleep apnea $(\mathrm{M})$, and severe sleep apnea $(\mathrm{S})$, were $0.67 \pm 0.19,0.63 \pm 0.11$ and $0.51 \pm 0.14$ respectively. These values are similar to our results except for subjects without sleep apnea who showed a scaling exponent of $\beta 2=0.84$. Peng [20] reported average scaling exponents of $\alpha 1=1.201 \pm 0.178$ and $\alpha 2=0.998 \pm 0.124$ for healthy subjects, and for congestive heart failure subjects, $\alpha 1=0.803 \pm 0.259$ and $\alpha 2=0.998 \pm 0.124$. Thus, the pathologic subjects exhibit a "reverse" crossover and it is possible to distinguish between the two groups.

We found double crossover points in the plot of $\log \mathrm{F}(\mathrm{n})$ vs. logn in patients with sleep apnea. In order to calculate the scaling exponent $\beta 2$, we set the first crossover point at $\mathrm{n}=50(\mathrm{CP} 1)$ and the second at $\mathrm{n}=251$ (CP2). Concerning exponent $\beta 2$, there was a rapid decrease of the slope after the first crossover point $(50<\mathrm{n}<250)$ and a rapid increase of the slope after the second $\mathrm{CP} 2$.

The amplitude and period of sinusoidal trends have an effect on the crossover point [26,27]. The period of a sinusoidal trend determines the crossover point [27]. Detrended fluctuation analysis for time sequential data obtained from the period of sinusoidal signal Xs with a period of $\mathrm{T}$ and amplitude $\mathrm{A}$ disclosed a crossover point of $\mathrm{T}$ beats and amplitude A leads to a vertical shift of $\log \mathrm{F}(\mathrm{n})$. After CP1, the slope decreases nearly flat (slope $=0$ ). Thus the cyclic change of the heartbeats with a cycle length of $\mathrm{T}$ beats observed in subjects with periodic breathing might produce a $\mathrm{CP} 1$ of $\mathrm{T}$ beats. These data are very similar to our results.

There are several sinusoidal rhythms of the heart beat 
in our pathophysiological system: 1) respiratory sinus arrhythmia (especially observed during deep sleep) with a cycle length of 4 to 8 seconds; 2$)$ the Mayer rhythm (10 seconds); 3) sleep apnea with a cycle length of $30-60$ seconds; and 4) Cheyne-Stokes respiration (cycle length of 60 - 120 seconds). We quantitatively analyzed the cycle length of periodic breathing in three different conditions and subjects [1], and found that: 1) for normal subjects under hypobaric pressure, the cycle length is $19.6 \pm 3.5$ seconds; 2) in patients with sleep apnea syndrome, the cycle length is $58.5 \pm 11.1$ seconds; and 3 ) in patients suffering from congestive heart failure accompanied by Cheyne-Stokes respiration, the cycle length is $93.2 \pm 21.7$ seconds. In the present study, we observed CP1 at 40 beats, indicating the cycle length of the sleep apnea. The rhythm regulation system in the central nervous system (CNS) has the ability to affect the sinus node of the heart through the autonomic nervous system, and may contribute to produce this crossover point [28].

This is the first study to point out the presence of CP2 in subjects suffering from sleep apnea. There was a statistically significant negative correlation between the number of apnea events and the value of scaling exponent $\beta 2(\mathrm{p}<0.05)$.

Further study might be required: 1) to clarify the physiological or mathematical significance of the presence of $\mathrm{CP} 2 ; 2$ ) to develop an algorithm to determine $\mathrm{CP} 1$ and $\mathrm{CP} 2$; and 3 ) to establish the sensitivity and specificity of $\beta 2$ in evaluating sleep apnea syndrome.

We conclude that the scaling exponent $\beta 2$ obtained from heartbeats detrended fluctuation analysis might be a useful parameter in evaluating the severity of sleep apnea.

\section{Acknowledgements}

We appreciate Roger G. Mark in MIT, J. W. Weiss and A. L. Goldberger in Beth Israel Deaconess Medical Center who kindly helped and organized us in recording and producing the MIT-BIH Polysomnographic database. None founding sources were used to obtain the results of the present study.

\section{REFERENCES}

[1] J. Motta and C. Guilleminault, "Cardiac Dysfunction during Sleep," Annals of Clinical Research, Vol. 17, 1985, pp. 190-198.

[2] K. Otsuka, Y. Ichimaru and T. Yanaga, "Studies of Arrhythmias by 24-Hour Polygraphic Recordings: Relationship between Atrioventricular Block and Sleep States," American Heart Journal, Vol. 105, No. 6, 1983, pp. 934940. doi:10.1016/0002-8703(83)90393-9

[3] Y. Ichimaru, Y. Kodama and T. Yanaga, "Circadian Variation of Heart Rate Variability," The Computer Society of the IEEE, Computers in Cardiology, Vol. 14, 1988, pp.
315-318

[4] H. Palomeki, "Snoring and the Risk of Ischemic Brain Infarction," Stroke, Vol. 22, 1991, pp. 1021-1025. doi:10.1161/01.STR.22.8.1021

[5] A. G. Tilkian, C. Guilleminault, J. S. Schroeder, et al., "Hymodynamics in Sleep Induced Apnea. Studies during Wakefulness and Sleep," Annals of Internal Medicine, Vol. 85, 1976, pp. 714-719.

[6] E. Lugaresi, G. Coccagna, F. Cirignotta, P. Farnetti, R. GaBassi, G. Di Donato, et al., "Breathing during Sleep in Man in Normal and Pathological Conditions," Advances in Experimental Medicine and Biology, Vol. 99, 1978, pp. 35-45. doi:10.1007/978-1-4613-4009-6 5

[7] C. Guilleminault, J. Van Den Hoed and M. M. Miller, "Clinical Overview of the Sleep Apnea Syndromes," In: C. Guilleminault and W. C. Dement, Eds., Sleep Apnea Syndromes, Alan R. Liss, Inc., New York, 1978, pp. 1-12.

[8] B. Buruch, C. Pollack, B. Borowiecki and E. Weitzman, "The Hypersomnia-Sleep Apnea Syndrome: A Reversible Major Cardiovascular Hazard," Circulation, 1977, Vol. 56, p. 177.

[9] R. P. Milkmen, S. Redline, C. C. Carlisle, A. R. Assaf and P. D. Levinson, "Day-Time Hypertension in Obstructive Sleep Apnea. Prevalence and Contributing Risk Factor," Chest, Vol. 99, No. 4, 1991, pp. 861-866. doi:10.1378/chest.99.4.861

[10] E. C. Fletcher, R. D. Debeholce, S. Lovoi and A. B. Garin, "Undiagnosed Sleep Apnea in Patients with Essential Hypertension," Annals of Internal Medicine, Vol. 103, 1985, pp. 190-195.

[11] A. J. Williams, D. Houston, S. Finberg, C. Lam, J. L. Kinney and S. Santiago, "Sleep Apnea Syndrome and Essential Hypertension," American Journal of Cardiology, Vol. 55, No. 8, 1985, pp. 1019-1022. doi:10.1016/0002-9149(85)90738-6

[12] M. Koskenvuo, J. Kaprio, T. Telakivi, M. Partinen, K. Heikkila and S. Sarna, "Snoring as a Risk Factor for Ischaemic Heart Disease and Stroke in Men," British Medical Journal, Vol. 294, 1978, pp. 16-19. doi:10.1136/bmj.294.6563.16

[13] A. R. Tilkian, C. Guilleminault, J. S. Schroeder, K. L. Lehrman, B. L. Simmons and W. C. Dement, "Sleep Induced Apnea Syndrome: Prevalence of Cardiac Arrhythmias and Their Reversal after Tracheostomy," American Journal of Medicine, Vol. 63, No. 3, 1977, pp. 348-358. doi:10.1016/0002-9343(77)90272-8

[14] C. Guilleminault, S. Connolly and R. Winkle, "Cardiac Arrhythmia and Conduction Disturbances during Sleep in 400 Patients with Sleep Apnea Syndrome," American Journal of Cardiology, Vol. 52, No. 5, 1983, pp. 490-494. doi:10.1016/0002-9149(83)90013-9

[15] A. Vazir, M. Dayer, P. C. Hasting, H. F. MiIntyre, et al., "Can Heart Rate Variation Rule out Sleep Disordered Breathing Heart Failure?" European Respiratory Journal, Vol. 27, No. 3, 2006, pp. 571-577, doi:10.1183/09031936.06.00130904

[16] C. Guilleminault, S. Connonly, R. Winkle, K. Melvin, et al., "A Cyclical Varation of the Heart Rate in Sleep Apnoea Syndrome, Mechanisms, and Usefulness of $24 \mathrm{~h}$ 
Electrocardiography as a Screening Technique," Lancet, Vol. 323, No. 8369, 1984, pp. 126-131. doi:10.1016/S0140-6736(84)90062-X

[17] Y. Ichimaru, M. Ichimaru, Y. Kodama and T. Yanaga, "Detection of Periodic Breathing during 24-Hour Ambulatory ECG Monitoring," IEEE Computers in Cardiology, Vol. 13, 1987, pp. 411-414.

[18] Y. Ichimaru and T. Yanaga, "Frequency Characteristics of the Heart Rate Variability Produced by Cheyne-Stokes Respiration during Ambulatory Monitoring," Computers and Biomedical Research, Vol. 22, No. 3, 1989, pp. 225233. doi:10.1016/0010-4809(89)90003-7

[19] F. Roche, E. Sforza, D. Duverney, et al., "Heart Rate Increment: An Electrocardiological Approach for the Early Detection of Obstructive Sleep Apnoea/Hypopnea Syndrome," Clinical Science, Vol. 107, 2004, pp. 105110. doi:10.1042/CS20040036

[20] C. K. Peng, S. Havlin, H. E. Stanley and A. L. Goldberger, "Quantification of Scaling Components and Crossover Phenomena in Nonstationary Heartbeat Time Series," Chaos, Vol. 5, No. 1, 1995, pp. 82-87. doi:10.1063/1.166141

[21] H. V. Huikiri, T. H. Makikallio, C. K. Peng, A. L. Goldberger, et al., "Fractal Correlation Properties of R-R Interval Dynamics and Mortality in Patients with Depressed Left Ventricular Function after an Acute Myocardial Infarction," Circulation, Vol. 101, 2000, pp. 47-53. doi:10.1161/01.CIR.101.1.47

[22] J. M. Tapanainen, P. E. Thomsen, L. Kober, C. TorpPedersen, T. H. Makikallio, A. M. Still, K. S. Lindgren and H. V. Huikuri, "Fractal Analysis of Heart Rate Vari- ability and Mortality after an Acute Myocardial Infarction," American Journal of Cardiology, Vol. 90, No. 4, 2002, pp. 347-352. doi:10.1016/S0002-9149(02)02488-8

[23] T. Penzel, J. W. Kantelhardt, L. Crote, J. H. Peter and A. Bundle, "Comparison of Detrended Fluctuation Analysis and Spectral Analysis for Heart Rate Variability in Sleep and Sleep Apnea," IEEE Transactions on Biological Engineering, Vol. 50, No. 10, 2003, pp. 1143-1151. doi:10.1109/TBME.2003.817636

[24] Y. Ichimaru and G. B. Moody, "Development of the Polysomnographic Database on CD-ROM," Psychiatry and Clinical Neurosciences, Vol. 53, No. 2, 1999, pp. 175-177. doi:10.1046/j.1440-1819.1999.00527.x

[25] A. Rechtschaffen and A. Kales, "A Manual of Standardized Terminology, Techniques and Scoring System for Sleep Stages of Human Sleep Subjects," US Government Printing Office, Washington DC, 1968.

[26] K. Hu, P. Ch. Ivanov, Z. Chen, P. Carpena and H. E. Stanley, "Effect of Trends on Detrended Fluctuation Analysis," Physical Review, Vol. 64, 2001, pp. 1-19.

[27] D. T. Schmitt and P. Ch. Ivanov, "Fractal Scale-Invariant and Nonlinear Properties of Cardiac Dynamics Remain Stable with Advanced Age: A New Mechanistic Picture of Cardiac Control in Healthy Elderly," American Journal of Physiology Regulatory, Integrative and Comparative Physiology, Vol. 293, No. 5, 2007, pp. R1923-R1937, doi:10.1152/ajpregu.00372.2007

[28] Y. Ichimaru and S. Katayama, "Rhythms in the Central Nervous System and 1/f Fluctuations of the Heart Rate," Frontiers of Medical \& Biological Engineering, Vol. 60, No. 2, 1994, pp. 117-130. 